\title{
Clozapine modulates retinoid homeostasis in human brain and normalizes serum retinoic acid deficit in patients with schizophrenia
}

\author{
Francesca Regen ${ }^{1}$ - Nicoleta-Carmen Cosma ${ }^{1}$ - Lisa R. Otto ${ }^{1}$. Vera Clemens ${ }^{1} \cdot$ Lana Saksone $^{1}$ - Janine Gellrich ${ }^{1}$. \\ Berk Uesekes $^{1} \cdot$ Thi Minh Tam Ta $^{1}$ - Eric Hahn ${ }^{1}$ - Michael Dettling ${ }^{1} \cdot$ Isabella Heuser ${ }^{1}$ Julian Hellmann-Regen $\mathbb{( I}^{1}$
}

Received: 12 December 2019 / Revised: 11 May 2020 / Accepted: 15 May 2020 / Published online: 2 June 2020

(c) The Author(s) 2020. This article is published with open access

\begin{abstract}
The atypical antipsychotic clozapine is one of the most potent drugs of its class, yet its precise mechanisms of action remain insufficiently understood. Recent evidence points toward the involvement of endogenous retinoic acid (RA) signaling in the pathophysiology of schizophrenia. Here we investigated whether clozapine may modulate RA-signaling. Effects of clozapine on the catabolism of all-trans RA (at-RA), the biologically most active metabolite of Vitamin A, were assessed in murine and human brain tissue and peripheral blood-derived mononuclear cells (PBMC). In patients with schizophrenia with and without clozapine treatment and matched healthy controls, at-RA serum levels and blood mRNA expression of retinoidrelated genes in PBMCs were quantified. Clozapine and its metabolites potently inhibited RA catabolism at clinically relevant concentrations. In PBMC-derived microsomes, we found a large interindividual variability of the sensitivity toward the effects of clozapine. Furthermore, at-RA and retinol serum levels were significantly lower in patients with schizophrenia compared with matched healthy controls. Patients treated with clozapine exhibited significantly higher at-RA serum levels compared with patients treated with other antipsychotics, while retinol levels did not differ between treatment groups. Similarly, in patients without clozapine treatment, mRNA expression of RA-inducible targets CYP26A and STRA6, as well as at-RA/retinol ratio, were significantly reduced. In contrast, clozapine-treated patients did not differ from healthy controls in this regard. Our findings provide the first evidence for altered peripheral retinoid homeostasis in schizophrenia and suggest modulation of RA catabolism as a novel mechanism of action of clozapine, which may be useful in future antipsychotic drug development.
\end{abstract}

\section{Introduction}

The atypical antipsychotic clozapine remains the first choice in treatment-resistant schizophrenia (SZ) [1-6]. Despite its potential to cause agranulocytosis/granulocytopenia and metabolic side effects, it exhibits extraordinary antipsychotic properties with negligible risk of extrapyramidal side effects [7]. Clozapine is known for its moderate binding affinity to

Supplementary information The online version of this article (https:// doi.org/10.1038/s41380-020-0791-8) contains supplementary material, which is available to authorized users.

Julian Hellmann-Regen

julian.hellmann@charite.de

1 Charité - Universitätsmedizin Berlin, corporate member of Freie Universität Berlin, Humboldt-Universität zu Berlin, and Berlin Institute of Health, Department of Psychiatry, Campus Benjamin Franklin, Berlin, Germany various neurotransmitter receptors while exhibiting a low affinity to the canonical D2 receptor [8]. These direct neurotransmitter-associated effects cannot sufficiently explain the remarkable efficacy of clozapine in treatment-refractory SZ. This implies that other non-neurotransmitter-associated effects may be expected to underlie its outstanding antipsychotic properties [9-12]. Moreover, both clozapine and its two major metabolites, clozapine-N-oxide (CNO) and $\mathrm{N}$-desmethylclozapine (NDC), exhibit neuroprotective and anti-inflammatory properties [13, 14].

Accumulating evidence suggests that disruption of synaptic functions, triggered at different stages during life, constitutes the neurobiological basis of adaptation deficits in the brain circuitry of patients with SZ [15]. These processes are regulated by paracrine retinoid signaling [16-18]. Several lines of evidence directly point toward disturbed retinoid signaling in schizophrenia [19-25]. All-trans retinoic acid (at-RA), the most active metabolite of vitamin A is locally produced and tightly regulated in brain tissue through 
cytochrome P450- (CYP450) mediated degradation into polar metabolites [26]. Retinoid signaling plays pivotal roles during embryonic development and neuronal differentiation $[27,28]$. In the adult brain, RA gradients between synthesizing and degrading sites of brain tissue also control several aspects of neuronal plasticity, including long term potentiation and depression (LTP/LTD) [29, 30], neuritogenesis [31], and the process of metaplasticity $[16,17]$. Metaplasticity is required for scaling synaptic strength within a neuronal network in a homeostatic manner and appears to be disturbed in SZ [18, 32-34], possibly due to dysregulated RA signaling in these patients [35]. In addition, evidence from hypothesisfree transcriptomic/proteomic analyses and genome-wide association data point toward altered retinoid signaling in the pathogenesis of SZ [21, 23, 36-38]. Finally, dopaminergic pathways are under direct transcriptional control of retinoids [39]. Based on these associations, first retinoid-targeting clinical trials using bexarotene, a retinoid-X-receptor (RXR)directed retinoid, show promising results in patients with SZ [40-42].

Based on evidence for dysregulated retinoid homeostasis in SZ, we hypothesized that some of the pleiotropic actions of clozapine might be mediated via direct effects on RA homeostasis. We hypothesized that clozapine might modulate RA homeostasis via direct interactions with local brain $a t$-RA catabolism, which is highly prevalent in the human brain and the most relevant regulatory step in brain at-RA signaling [43-45]. To identify possible effects of clozapine and its metabolites on retinoid homeostasis, we used a previously established methodology to assess tissue-specific catabolism of RA in murine tissues, human postmortem brain tissue and human peripheral blood-derived mononuclear cells (PBMC) in vitro [46-48]. Furthermore, we assessed various RA homeostasis-related parameters in an observatory clinical study of patients with SZ with and without clozapine medication and matched healthy controls.

\section{Materials, patients, and methods}

\section{Materials}

All chemicals were purchased from Sigma-Aldrich (Taufkirchen, Germany) unless otherwise stated.

\section{Cell culture and tissue preparation}

\section{Serum and PBMC isolation}

For a detailed description of serum and PBMC isolation, please refer to the Supplement S1. In brief, whole blood was collected in the respective vacuum-extraction tubes and prepared according to the manufacturer's instructions.
Aliquots were stored at $-80{ }^{\circ} \mathrm{C}$. For PBMC isolation, heparinized blood was extracted by FICOLL ${ }^{\mathrm{TM}}$ density gradient centrifugation, following previously published protocols [49].

\section{Preparation of crude microsomal and synaptosomal fractions for RA metabolism assays}

Human postmortem brain tissue from the superior temporal gyrus of five healthy donors was obtained from the Netherlands Brain Bank (NBB, Netherlands Institute for Neuroscience, Amsterdam). All donors gave written informed consent for brain autopsy and for the use of specimens for research purposes. Mouse tissue was derived from C57/BL6, P0-P3, male and female animals. Animals were sacrificed by decapitation before tissue preparation. All experiments including animals were registered and approved by German regulatory authorities (T 0268/15) and approved by a local institutional review board.

CYP450-containing, metabolically active crude microsomal and synaptosomal fractions from brain tissues and isolated PBMCs were prepared according to previously published protocols with minor modifications $[48,50]$. For a detailed description of the procedures, please refer to the Supplementary S1. In brief, tissues or cells from healthy donors (Supplementary Table 1) were homogenized and metabolically active fractions were prepared by differential centrifugation steps. Metabolically active crude synaptoso$\mathrm{mal} /$ microsomal fractions were stored at $-80^{\circ} \mathrm{C}$, protein concentrations were determined by the BCA method (Thermo Fisher, USA).

\section{RA catabolism assay}

In vitro assays to quantify RA metabolism were performed as previously published [51, 52]. A more detailed description of the methods is available in Supplementary S1. In brief, samples contained metabolically active enzyme preparations, the test compound (drug) at the desired final concentration, RA $(1 \mu \mathrm{M}), \mathrm{NADPH}(800 \mu \mathrm{g} / \mathrm{ml})$ and assay buffer. Heat-inactivated controls were incubated at $95{ }^{\circ} \mathrm{C}$ for $15 \mathrm{~min}$. Reactions were allowed to incubate for $60 \mathrm{~min}$ at $37^{\circ} \mathrm{C}$ and were stopped by the addition of ice-cold methanol. Subsequently, all samples were centrifuged at $21,000 \times g, 4{ }^{\circ} \mathrm{C}$ and subjected to retinoid analysis. All steps were carried out under dim, yellow light. RA metabolic activity was calculated by comparing RA degradation in metabolically active samples with heat-inactivated controls.

\section{Participants}

Healthy donors and SZ patients within the clinical observational study on RA homeostasis in neuropsychiatric disorders 
(RAHND; ClinicalTrials.gov Identifier: NCT02439099) were included. The local ethics committee approved the study (EA4/002/13). All patients had a clinical DSM-5 diagnosis of SZ more than five years prior to inclusion were treated in our clinic as in- or outpatients and were on a stable medication with clozapine $(N=10)$ or other antipsychotics $(N=10$; Supplementary Table 2). All participants were matched for age, weight, BMI, and smoking status, including matched healthy controls $(N=10$; Table 1$)$. Participants arrived at the laboratory between 8 and 12 a.m. after an overnight fast for blood collection. Each participant provided a total volume of $40 \mathrm{ml}$ of peripheral venous blood.

\section{Serum extraction of retinoids}

The extraction of retinoids from human sera was performed by a liquid-liquid extraction procedure using the synthetic retinoid acitretin as an internal standard to assess recovery and account for inter- and intra-assay variability. Liquid-liquid extraction was performed by spiking fractions of $1 \mathrm{ml}$ of serum with internal standard (Acitretin) dissolved in DMSO, then adding $1 \mathrm{vol}$ of acidified ethanol containing $3 \%(\mathrm{v} / \mathrm{v})$ orthophosphoric acid to 1 vol of patient serum. Samples were vortexed for $1 \mathrm{~min}$ and 2 vol of hexane was added. Samples were vigorously vortexed for $15 \mathrm{~min}$ and centrifuged at $1560 \times g$ at $4{ }^{\circ} \mathrm{C}$ for $5 \mathrm{~min}$. The supernatant was evaporated to dryness under a gentle stream of Argon. Samples were resuspended in $1 \mathrm{ml}$ of HPLC running buffer.

\section{High-performance liquid chromatography}

High-performance liquid chromatography (HPLC) was performed as previously described [52] and specified in detail in Supplementary S1. In brief, RA isomers and RA degradation products were quantified using an Agilent 1100-series HPLC

Table 1 Participant characteristics.

\begin{tabular}{llll}
\hline & \multicolumn{2}{l}{ Schizophrenic patients } & \multicolumn{2}{l}{$\begin{array}{l}\text { Healthy } \\
\text { controls }\end{array}$} \\
\cline { 2 - 3 } & Clozapine & $\begin{array}{l}\text { Other } \\
\text { medication }\end{array}$ & \\
& $N=10$ & $N=10$ & $N=10$ \\
\hline Age (years \pm SD) & $42.2 \pm 11.1$ & $42.5 \pm 13.9$ & $42.2 \pm 11.3$ \\
Male/female $(n)$ & $5 / 5$ & $5 / 5$ & $5 / 5$ \\
BMI \pm SD & $25.2 \pm 5.0$ & $27.3 \pm 5.1$ & $25.9 \pm 3.9$ \\
Current smokers $(n)$ & 6 & 6 & 6 \\
PANSS total & $72.5 \pm 21.2$ & $86.6 \pm 18.1$ & NA \\
score \pm SD & & & \\
Years of medication $^{\mathrm{a}}$ & $15.3 \pm 9.9$ & $4.5 \pm 3.9$ & NA
\end{tabular}

BMI Body Mass Index, PANSS Positive and Negative Syndrome Scale, $N A$ not applicable.

${ }^{a}$ Significantly different from healthy controls. system equipped with a Supelco Suplex ${ }^{\oplus}$ column $(5 \mu \mathrm{m}, 2.1 \times$ $250 \mathrm{~mm}$; for pharmacological assays) or a Phenomenex Synergi RP $4 \mu \mathrm{m} 80 \mathrm{~A}$ column (for serum analyses) and a 1260 -series diode-array detector with UV detection at $340 \mathrm{~nm}$ for at-RA detection and $320 \mathrm{~nm}$ for retinol (ROL) detection. Peaks were identified by authentic standards and purity was routinely checked by online spectral analysis.

\section{Real-time PCR}

Blood was collected in PAXgene tubes (PreAnalytiX $\mathrm{GmbH}$ ) and RNA was extracted following the manufacturer instructions. Total RNA was then reverse transcribed into cDNA using Revert Aid First Strand cDNA Synthesis Kit ${ }^{\mathrm{TM}}$ (Thermo Fisher Scientific Inc., MA, USA). All primers were designed and checked for their quality using the PrimerBLAST software [53]. Primer sequences and further details on qPCR analyses are available in the Supplementary S1.

\section{Statistical analyses}

Numerical analyses were performed using GraphPad statistical software version 5.04 (GraphPad Software, La Jolla, USA). For inhibition characteristics of RA catabolism by clozapine, 3- or 4-parameter nonlinear regression analysis was performed using GraphPad Prism. Values for maximum inhibition and half-maximal inhibitory concentrations (IC50) were calculated based on the regression analysis. Differences between groups were investigated by Student's $t$ test, one-way analysis of variance (ANOVA) with Newman-Keuls multiple comparisons test or Kruskal-Wallis test with Dunn's multiple comparisons test when appropriate. $P$ values $<0.05$ were considered statistically significant. Values are given as mean \pm S.E.M. $* P<0.05$, ** $P<0.01$, *** $P<0.001$.

\section{Results}

\section{Clozapine blocks RA catabolism in murine whole brain}

In mouse whole brain-derived synaptosomal fractions, clozapine strikingly blocks RA catabolism, starting at concentrations as low as $1 \mu \mathrm{M}$ when assessed by either reduction of $a t$-RA levels or synthesis of the $a t$-RA degradation product 4-oxo-RA (Fig. 1a, b).

The representative chromatograms (Fig. 1a) demonstrate low to absent RA catabolism in heat-inactivated samples and substantial RA degradation in active samples by means of reduced at-RA peaks and increased RA metabolites. Upon addition of clozapine at 5 or $50 \mu \mathrm{M}$, at-RA peaks in active synaptosomes increase, while peaks of the RA metabolite 4-oxo-RA decrease in a concentration-dependent 
A

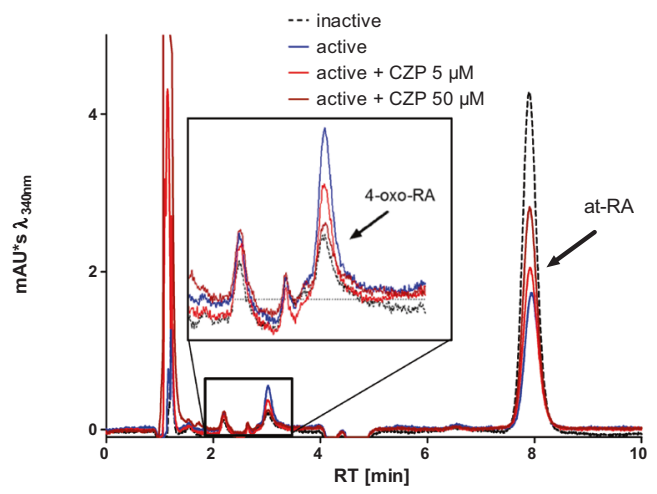

c

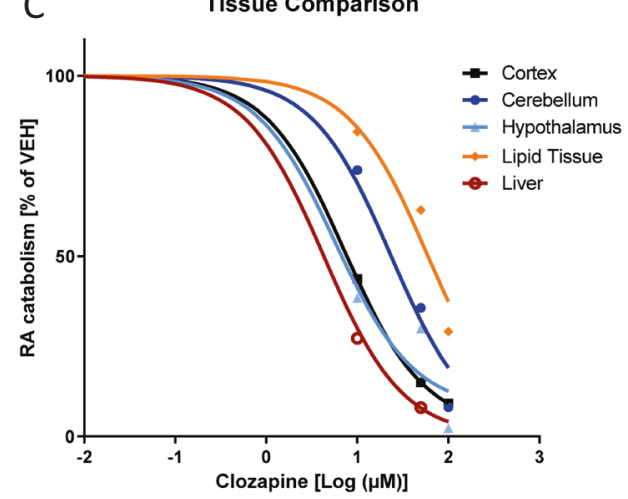

Fig. 1 Clozapine blocks RA degradation in murine tissues. a HPLC Chromatograms of at-RA catabolism assays from representative samples containing heat-inactivated (blue line) or active crude synaptosomal fractions from mouse brain exposed to at-RA at $1 \mu \mathrm{M}$ and treated either with vehicle (black, dashed line) or clozapine at 5 and $50 \mu \mathrm{M}$ (red lines). b Levels of at-RA (solid line) and its polar metabolite 4-OXO-RA (dashed line) following in vitro incubation $\left(1 \mathrm{~h}, 37^{\circ} \mathrm{C}\right)$ with murine brain-derived synaptosomes and at-RA $1 \mu \mathrm{M}$ starting concentration in the presence of various clozapine concentrations. Values are calculated relative to heat-inactivated and vehicle-treated controls. Clozapine significantly inhibits at-RA degradation in a dose-dependent manner, blocking on average $>80 \%$ of at-RA catabolism in microsomal

manner, indicating that clozapine potently blocks RA catabolism.

Figure $1 \mathrm{~b}$ further demonstrates the strong inhibitory effect of various clozapine concentrations on RA degradation when measured either by quantification of at-RA concentration or by measuring the increase in the concentration of RA metabolites (4-oxo-RA). The half-maximal inhibitory concentrations (IC50 value) for clozapine were $7.9 \mu \mathrm{M}$ as determined by measuring the reduction in at-RA and $7.8 \mu \mathrm{M}$ as determined by measuring the synthesis of 4-oxo-RA.

Local brain levels of clozapine that are expected to be reached when clozapine is given at therapeutically relevant doses [54] are indicated in the figures by means of rectangles labeled "brain tissue levels". These levels were calculated based on observations by Wilk et al. and

\section{B}
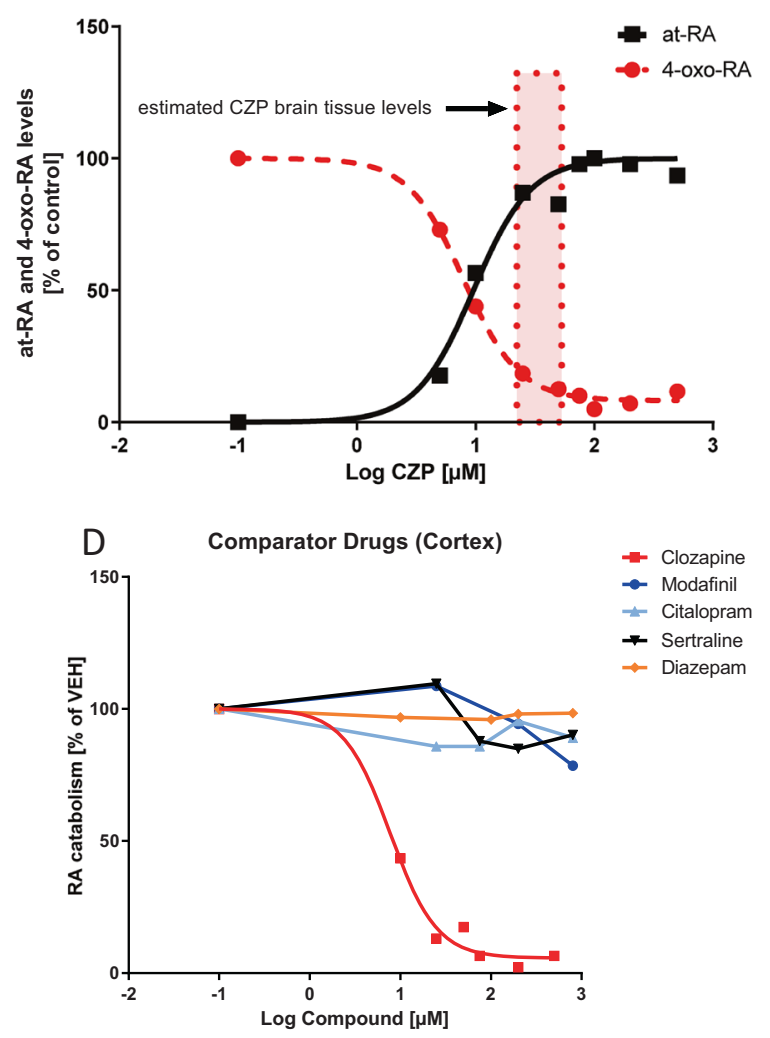

preparations, as calculated by 4-parameter nonlinear regression analysis. The dotted rectangle demonstrates the brain tissue levels of clozapine reached during steady state. c at-RA catabolism was measured by quantifying the total $a t$-RA turnover in clozapine-treated murine tissue samples relative to vehicle-treated samples. Different murine brain regions and other tissues exhibit differential sensitivity toward clozapine. d Various other psychotropic drugs were assessed in the same pharmacological assays, including the stimulant modafinil, the two antidepressants citalopram and sertraline and the benzodiazepine diazepam. Neither of the tested drugs exhibited inhibitory effects comparable to clozapine. All assays were performed at least in duplicates using pooled synaptosomes/microsomes from $N=3$ animals.

particularly Baldessarini et al., who measured both serum and brain clozapine levels in rats at steady state after intraperitoneal administration [54, 55]. While serum levels in rats reached levels comparable to human serum concentrations, lipophilic clozapine is preferentially distributed to brain tissue resulting on average in 24-fold higher brain concentrations compared with serum levels [54]. In humans, serum clozapine concentrations between 350 and $600 \mathrm{ng} / \mathrm{ml}$ are considered to be associated with an optimal therapeutic response [56]. Therefore, brain tissue levels of $8400-14400 \mathrm{ng} / \mathrm{ml}$, which equals $25.7-44.05 \mu \mathrm{M}$, are expected.

The increase in at-RA and the decrease in the synthesis of 4-oxo-RA in response to clozapine exposure were significantly correlated (Pearson's $r=0.990, P<0.001$ ), 
suggesting that measuring either the decrease of at-RA or the increase of 4-oxo-RA may be adequate to assess RA catabolism.

\section{Clozapine blocks RA catabolism in various murine tissues}

As we were interested in local differences of the effects of clozapine on RA catabolism and because of the known effects of clozapine on energy homeostasis with weight gain as one of the prominent side effects of the drug, we also assessed the impact of clozapine on RA catabolism in murine cerebellum, hypothalamus and in non-neuronal tissues (liver tissue and lipid tissue from visceral fat; Fig. 1c). Clozapine exhibited a differential impact on RA catabolism with most pronounced effects in liver (IC50 $=4.44 \mu \mathrm{M}$ ), cortex $($ IC50 $=7.30 \mu \mathrm{M})$ and hypothalamus (IC50= $5.76 \mu \mathrm{M})$ and less pronounced effects in cerebellum (IC50 $=54.12 \mu \mathrm{M})$ and in lipid tissue $($ IC50 $=699.5 \mu \mathrm{M})$.

\section{Impact of other psychotropic drugs on cerebral RA catabolism}

In order to compare the observed effects of clozapine on RA catabolism with other psychotropic drugs, we next tested the effects of the antidepressants citalopram and sertraline, the benzodiazepine diazepam and the stimulant modafinil on RA catabolism, using equally high concentrations, including concentrations above therapeutically relevant tissue levels (see Supplementary Table 3 for estimated brain concentrations) [57-61]. Neither of the tested compounds affected the degradation of RA in mouse cortex-derived synaptosomes to the extent of clozapine (Fig. 1d).

\section{Effects of clozapine on human tissues}

Next, we analyzed the impact of clozapine on RA catabolism in human PBMC-derived microsomes from eight healthy donors (Fig. 2; for participant characteristics see Supplementary Table 1). Clozapine strikingly inhibited RA catabolism in all samples (Fig. 2a), yet the degree of inhibition varied considerably between subjects. All subjects exhibited IC50 values (mean $12.5 \mu \mathrm{M}, \pm 9.2$ ) within or even below the estimated brain tissue concentrations of clozapine (Fig. 2a, b). The sensitivity of RA catabolism toward inhibition by clozapine (IC50) also differed markedly between subjects (Fig. 2b). While five out of eight subjects exhibited high sensitivity with IC50 values well below the calculated mean of $12.5 \mu \mathrm{M}$, there were three subjects with IC50 values markedly above the mean of $12.5 \mu \mathrm{M}$ (Fig. 2b). For five out of eight subjects, inhibition was predicted to result in $100 \%$ inhibition of RA catabolism (Fig. 2c).
Finally, we assessed RA catabolism in pooled metabolically active synaptosomes from human postmortem brain tissue of five healthy donors (Fig. 2d). Pooled synaptosomes, all prepared from the superior temporal gyrus of the donors, exhibited strong RA catabolic activity. At-RA degradation was also strikingly affected by clozapine with an IC50 value of $12.9 \mu \mathrm{M}$ (Fig. 2d), which is almost identical to the average IC50 value observed for human PBMCs (Fig. 2a) and comparable to murine brain (Fig. 1a).

\section{Clozapine metabolites N-Desmethylclozapine and Clozapine-N-oxide also affect at-RA catabolism}

The two major metabolites, "pharmacologically inert" CNO and NDC, both exhibit neuroprotective and antiinflammatory properties via unknown mechanisms, starting at concentrations as low as $0.01 \mu \mathrm{M}$ for $\mathrm{CNO}$ and $1 \mu \mathrm{M}$ for NDC [13]. Interestingly, both metabolites exhibit RA catabolism-blocking properties in murine cortex, pooled human PBMCs and human cortex (Fig. 3). Significant inhibition occurs at low micromolar concentrations that are expected to be reached in clinical practice. The strongest inhibition of RA metabolism was seen for both NDC $(\mathrm{IC} 50=0.40 \mu \mathrm{M})$ and $\mathrm{CNO}(\mathrm{IC} 50=2.9 \mu \mathrm{M})$ in pooled human PBMC-derived microsomes (Fig. 3c, g).

\section{Effects of clozapine on serum at-RA levels and PBMC mRNA transcripts of RA-related genes in patients with schizophrenia}

To assess clinical relevance for the observed effects of clozapine on RA catabolism, we measured for the first time atRA concentration in the serum of patients with SZ who had been on clozapine medication for more than five years (clozapine group), matched patients with SZ taking antipsychotics others than clozapine (no-clozapine group, Supplementary Table 2), and matched healthy controls (Table 1).

We found significantly reduced serum levels of $a t$-RA in patients with SZ compared with matched healthy controls $(P<0.0001$; Fig. 4a). In patients with SZ, at-RA serum levels were lowest in the no-clozapine group of patients with SZ $(2.2 \pm 1.2 \mathrm{nM})$ and significantly higher in the clozapine group $(3.7 \pm 1.3 \mathrm{nM} ; P<0.05)$. Both treatment groups exhibited lower at-RA levels when compared with healthy controls $(7.1 \pm 1.8 \mathrm{nM} ; P<0.001)$.

ROL levels were also significantly reduced in patients with SZ of the no-clozapine group $(1.37 \pm 0.19 \mu \mathrm{M}, P<$ $0.001)$ and the clozapine group $(1.27 \pm 0.09 \mu \mathrm{M}, P<0.001)$ compared with healthy controls $(2.46 \pm 0.43)$, yet without the effect of medication (Fig. 4b).

Finally, the ratio of at-RA to ROL was calculated for each subject (Fig. 4c). Interestingly, ratios were significantly reduced only in the no-clozapine group $(1.64 \pm 0.16 \mathrm{nM} / \mu \mathrm{M})$ 
A

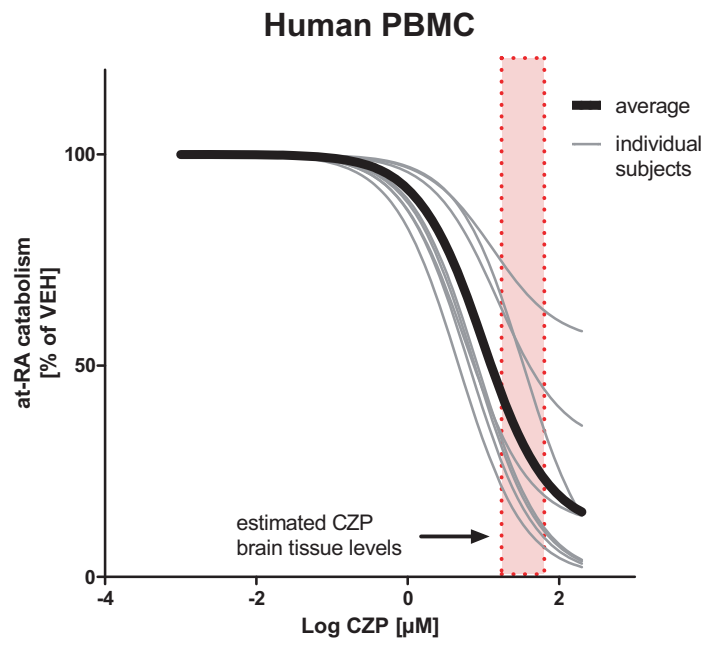

C

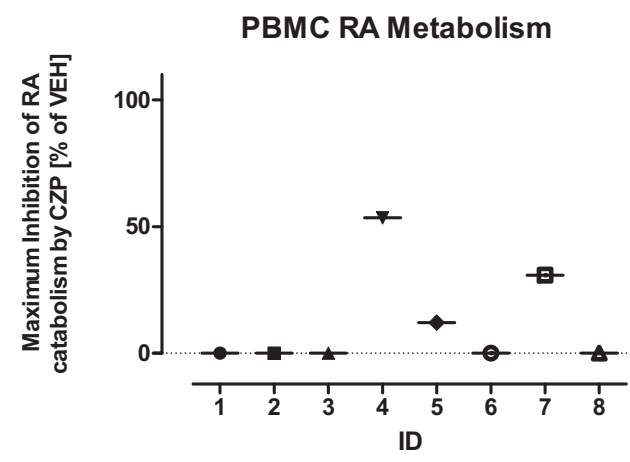

Fig. 2 Clozapine blocks at-RA degradation in human tissues. a Effects of clozapine on at-RA degradation in PBMC-derived microsomal preparations were assessed using cells from 8 healthy donors. Clozapine significantly blocked at-RA turnover in all subjects (lightweight curves) with an average IC50 value of $9.86 \mu \mathrm{M}$ (dark bold curve). b IC50 values and c maximum inhibitability, as calculated by 4-parameter nonlinear regression, exhibited significant variability

when compared with healthy controls $(2.88 \pm 0.28 \mathrm{nM} / \mu \mathrm{M}$; $P<0.001)$ and subjects from the clozapine group $(2.88 \pm$ $0.20 \mathrm{nM} / \mu \mathrm{M} ; P<0.001)$. Ratios in the clozapine group did not differ from healthy controls.

PBMC mRNA transcript levels of the RA catabolic enzyme CYP26A (Fig. 4d) and the RA-inducible protein STRA6 (Fig. 4f) both exhibited a similar profile as observed for the $a t$-RA/ROL ratio (Fig. 4c). Here, we found significantly lower CYP26A levels in the no-clozapine group of patients with SZ $(0.55 \pm 0.17)$ compared with healthy controls $(1.0 \pm 0.33 ; P<0.05)$ or subjects treated with clozapine $(0.85 \pm 0.27 ; P<0.05)$, while there was no difference between clozapine-treated patients and healthy controls. In contrast, there were no main effects of group for the RA-synthesizing enzyme aldehyde dehydrogenase 1A2 (ALDH1A2; Fig. 4e).

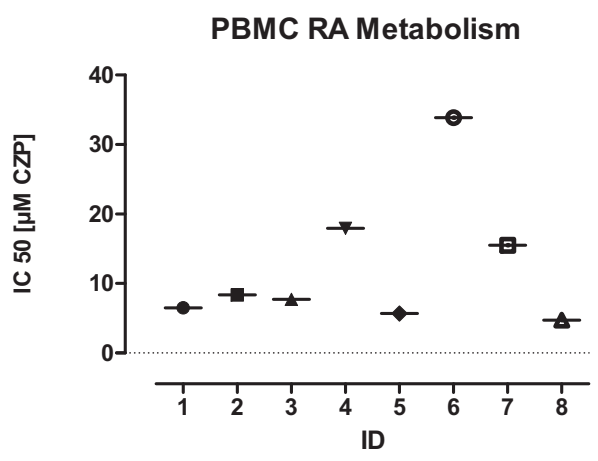

Human Temporal Cortex

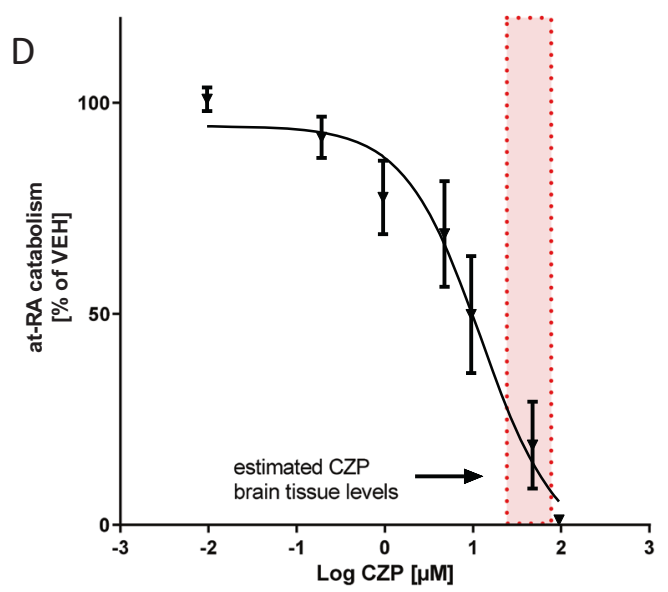

between the 8 subjects. d Clozapine also significantly blocks at-RA degradation in pooled human brain-derived synaptosomal preparations derived from postmortem tissue from 5 healthy subjects (IC50: 12.9 \pm $1.56 \mu \mathrm{M})$. The dotted rectangle demonstrates the brain tissue levels of clozapine during steady state. Values are given as mean and standard error of $n \geq 3$ experiments.

We also assessed mRNA expression of the retinoic acid receptor (RAR) and RXR $\alpha, \beta$ and $\gamma$ isotypes (Fig. 5). While RAR- $\gamma$ was not detectable, there were no significant main effects of group for any of the receptors. Finally, mRNA expression of non-specific RA-degrading cytochrome isoforms CYP1A2, 2D6, and 3A4 were quantified in PBMCs, exhibiting significantly reduced expression of CYP2D6 and 3A4 in clozapine-treated subjects (Fig. $4 \mathrm{f}-\mathrm{h}$ ).

\section{Discussion}

We demonstrated for the first time that clozapine and its major metabolites specifically inhibit $a t$-RA catabolism in a dose-dependent and clinically relevant manner. Furthermore, we provide clinical evidence on significantly reduced 

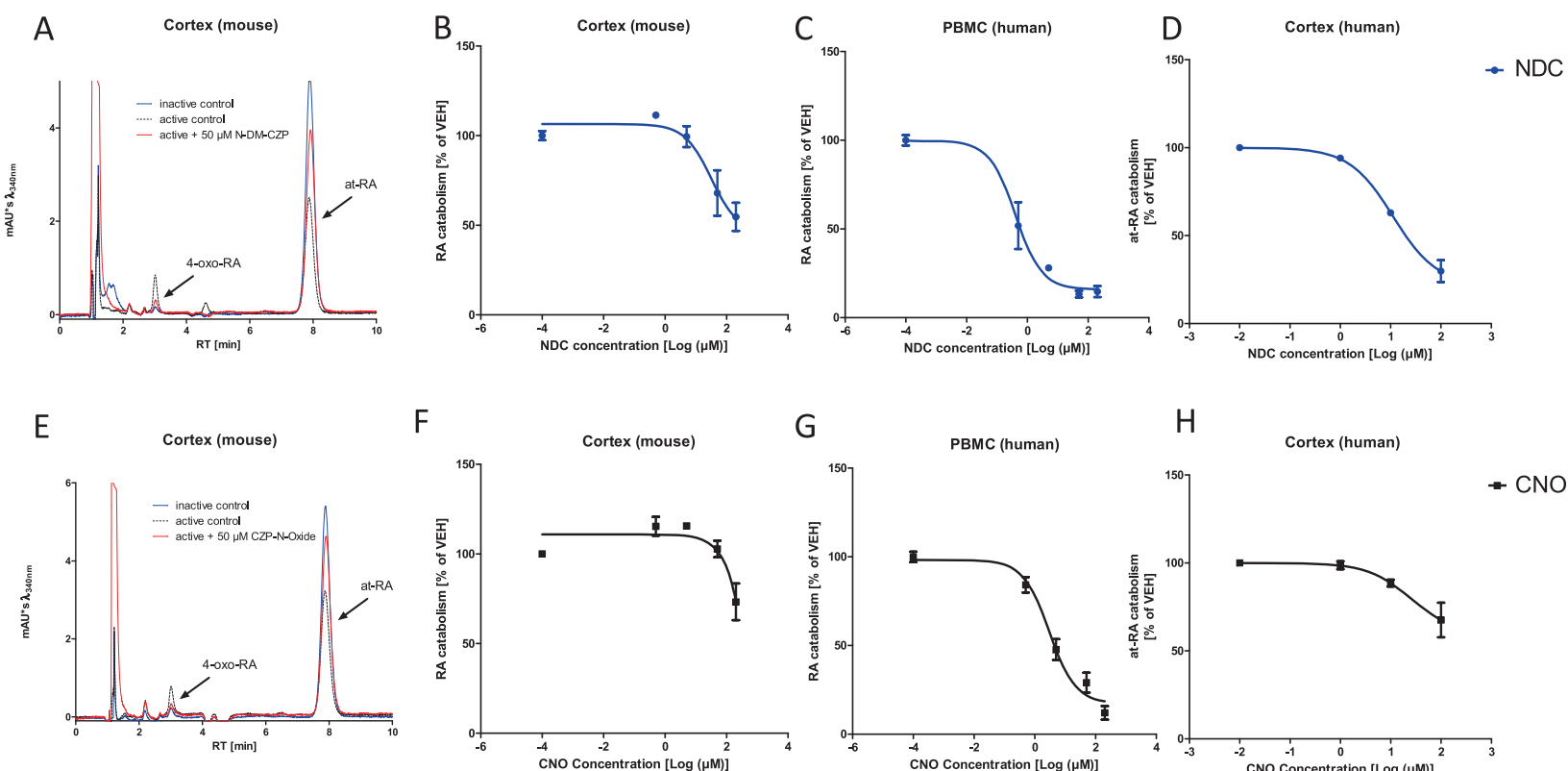

G

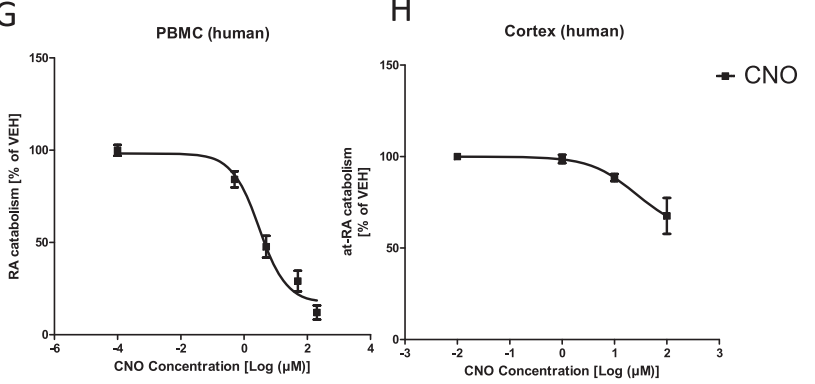

Fig. 3 Impact of Clozapine Metabolites on at-RA Catabolism. a Representative chromatographs of at-RA catabolism in murine cortex demonstrate the impact of N-Desmethylclozapine (NDC) on $a t$-RA degradation. NDC significantly blocks at-RA catabolism in synaptosomes derived from mouse cortex (b), human PBMCs (c) and human cortex (d). Clozapine-n-oxide (CNO) also exhibits significant,

retinoid serum levels in patients with schizophrenia and on clozapine positively modulating RA homeostasis in clozapine-treated patients.

Our findings are in line with the "retinoid hypothesis of schizophrenia" [35], which is based on several fundamental findings. First, endogenous retinoids are crucially involved in maintaining neuronal homeostasis by acting as potent endogenous neuroprotective compounds and regulators of inflammation, particularly microglial activation [62, 63]. Moreover, retinoid signaling is increasingly recognized as a key mediator in metaplasticity [16], which is suggested to be disturbed in SZ [34, 64].

In line with the hypothesis of deranged metaplastic processes during the course of illness, several other lines of evidence have also suggested dysregulated retinoid homeostasis in the pathophysiology of schizophrenia [40]. While early studies have clearly identified retinoid dysregulation at the transcriptome level in patients with SZ [37], there is also more recent genome-wide proof for retinoid dysregulation in SZ [21]. Moreover, evidence from retinoid-based treatments supports the hypothesis that specific retinoid-targeting interventions may serve as novel treatment options [41, 42].

When we think about retinoid-based interventions to treat e.g. patients with SZ, it is important to consider that the use of exogenous retinoids may be somewhat compromised by homeostatic adaptations within the locally controlled retinoid signaling network (e.g., induction of local degradation, downregulation of endogenous synthesis) [65].

yet less pronounced inhibition in murine cortex $(\mathbf{e}, \mathbf{f})$, human PBMCs (g) and human cortex (h) with IC50 values within the expected brain tissue levels according to 4-paramter nonlinear regression analysis. All experiments were performed at least in duplicates and values are given as mean and standard error.

To overcome this problem, it may be an option to enhance retinoid signaling by means of blocking RA degradation, which is a pivotal step in controlling local RA levels $[28,65,66]$. We previously identified this mechanism for pleiotropic minocycline [46, 48, 67], a tetracycline antibiotic and modulator of microglial activity discussed for use in SZ [68-70]. Out of all antipsychotics available, clozapine is still considered the most effective drug in treatmentrefractory SZ. Nevertheless, its precise mechanism of action remains unclear. Based on our findings for minocycline and the fact that catabolism of clozapine involves RA-degrading CYP450 enzymes, we hypothesized clozapine to functionally block endogenous RA catabolism. Further support for this hypothesis comes from a partial overlap of the side effects of clozapine and RA, which include dyslipidemia and impaired bone marrow functions, particularly reduced white blood-cell counts [71, 72].

To assess the effects of clozapine on RA degradation, we functionally measured $a t$-RA catabolism in various target tissues in the presence of various clozapine doses. While effects on RA catabolism were expected, the extent to which low, physiological clozapine concentrations blocked at-RA degradation was rather unexpected, and so were our findings on NDC and CNO, which equally affect at-RA degradation, thereby possibly explaining their pleiotropic effects [13].

Using RA-metabolizing microsomes of donor-derived PBMCs, we provide a potentially valuable tool to quantify a 

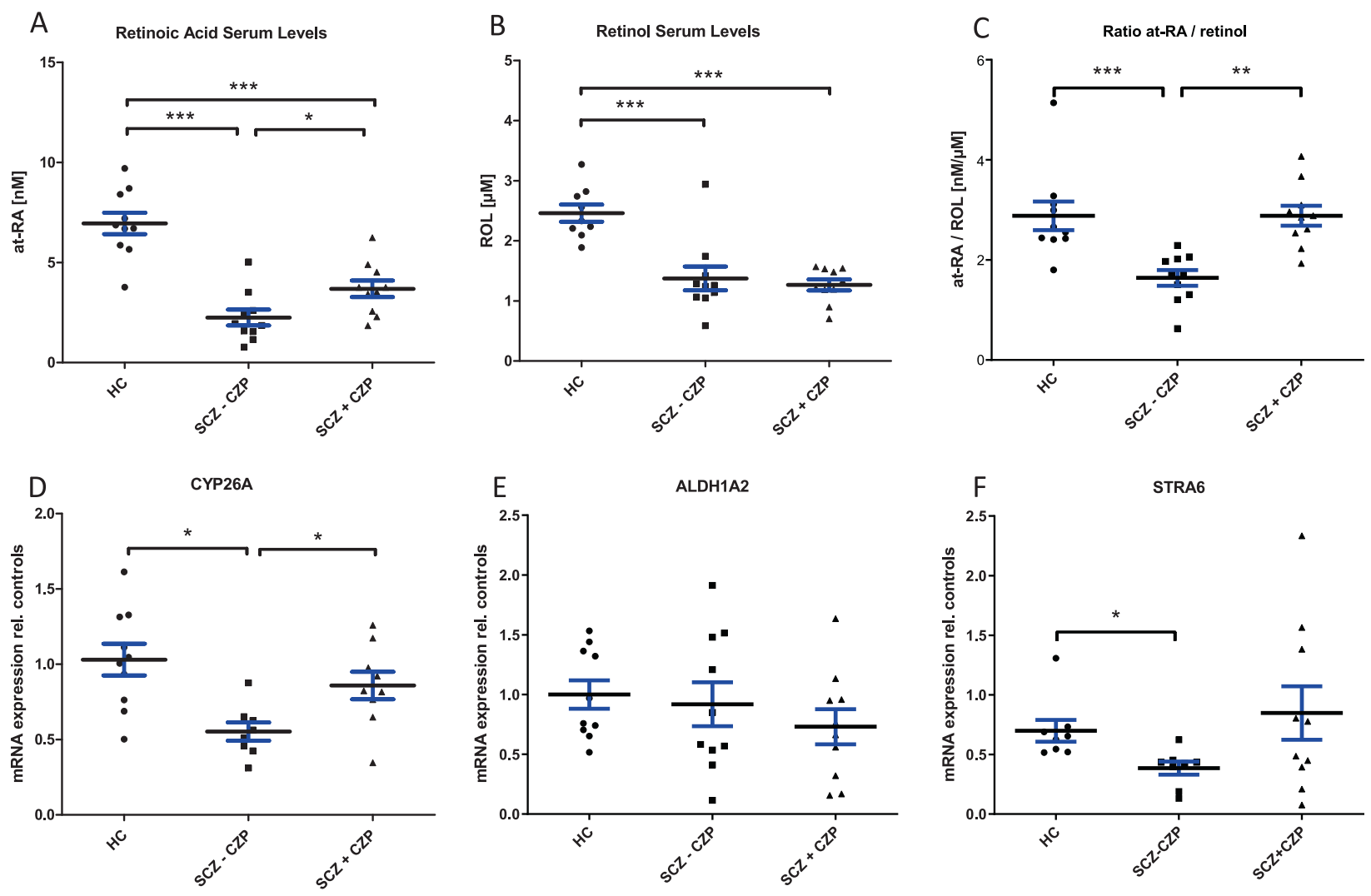

Fig. 4 Retinoid Homeostasis in Patients with Schizophrenia with and without Clozapine. a Retinoic acid serum levels were measured in healthy controls (HC; $N=10$ ), patients with schizophrenia receiving antipsychotic medication other than clozapine (SCZ - CZP; $N=$ 10) and patients with schizophrenia currently receiving clozapine $(\mathrm{SCZ}+\mathrm{CZP} ; N=10)$. Serum at-RA levels were significantly reduced in patients with schizophrenia compared with controls regardless of medication $(* * * P<0.0001$, one-way ANOVA with Newman-Keul's post-hoc test). at-RA levels in clozapine-treated patients were significantly increased compared with no-clozapine patients $(* P<0.05$, one-way ANOVA with Newman-Keul's post-hoc test). b Serum retinol (ROL) levels were significantly reduced in patients with

schizophrenia from either medication group compared with HC $(* * * P<0.0001)$. c The at RA to ROL ratio was significantly reduced only in the SCZ-CZP group. d Whole blood mRNA expression of $a t$-RA-catabolizing and $a t$-RA-inducible CYP26A was also strikingly lowered only in $\mathrm{SCZ}-\mathrm{CZP}$ subjects while there were no differences between $\mathrm{HC}$ and $\mathrm{SCZ}+\mathrm{CZP}(* P<0.05$, one-way ANOVA with Newman-Keul's post-hoc test). e, f mRNA levels of the RAsynthesizing enzyme ALDH1A2 did not differ between groups, while mRNA levels of RA-inducible gene STRA6 were significantly decreased in SCZ - CZP compared with healthy controls $(* P<0.05$, one-way ANOVA with Newman-Keul's post-hoc test).

patient-specific response of retinoid homeostasis to clozapine. While our study cannot directly answer the question of whether enhanced retinoid signaling may causally underlie clozapine's antipsychotic mode of action, our approaches are suitable to correlate the individual retinoid response to clozapine with clinical response in a longitudinal approach, which will be investigated prospectively in future clinical trials.

Retinoid dysregulation in SZ is a convincing theory; however, evidence on functionally altered retinoid signaling in SZ remains associative [19, 21, 25, 35]. We, therefore, assumed alterations in peripheral serum levels of at-RA to reflect an overall disturbed retinoid signaling.

Based on our finding that clozapine strikingly affects local at-RA degradation we established a three-group design including patients with SZ on an established drug regimen with clozapine ( $>5$ years continuous exposure), patients with SZ taking other antipsychotic drugs and healthy controls, all matched for age, gender, BMI and smoking status. Using a highly sensitive HPLC-based method [50,73] we were able to identify significantly reduced at-RA as well as ROL serum levels, reduced $a t$-RA/ROL ratios as well as reduced expression of the RA-inducible and RA-metabolizing CYP26A and the RAinducible protein stimulated by retinoic acid 6 (STRA6) in schizophrenia patients without clozapine treatment (Fig. 4). Furthermore, confirming our in vitro data, both at-RA serum levels, at-RA/ROL ratios, and CYP26-levels were increased in patients on clozapine compared with patients on other antipsychotics. Interestingly, PBMC mRNA expression of the RA-synthesizing enzyme ALDH1A2, for which genetic and epigenetic associations with SZ were 

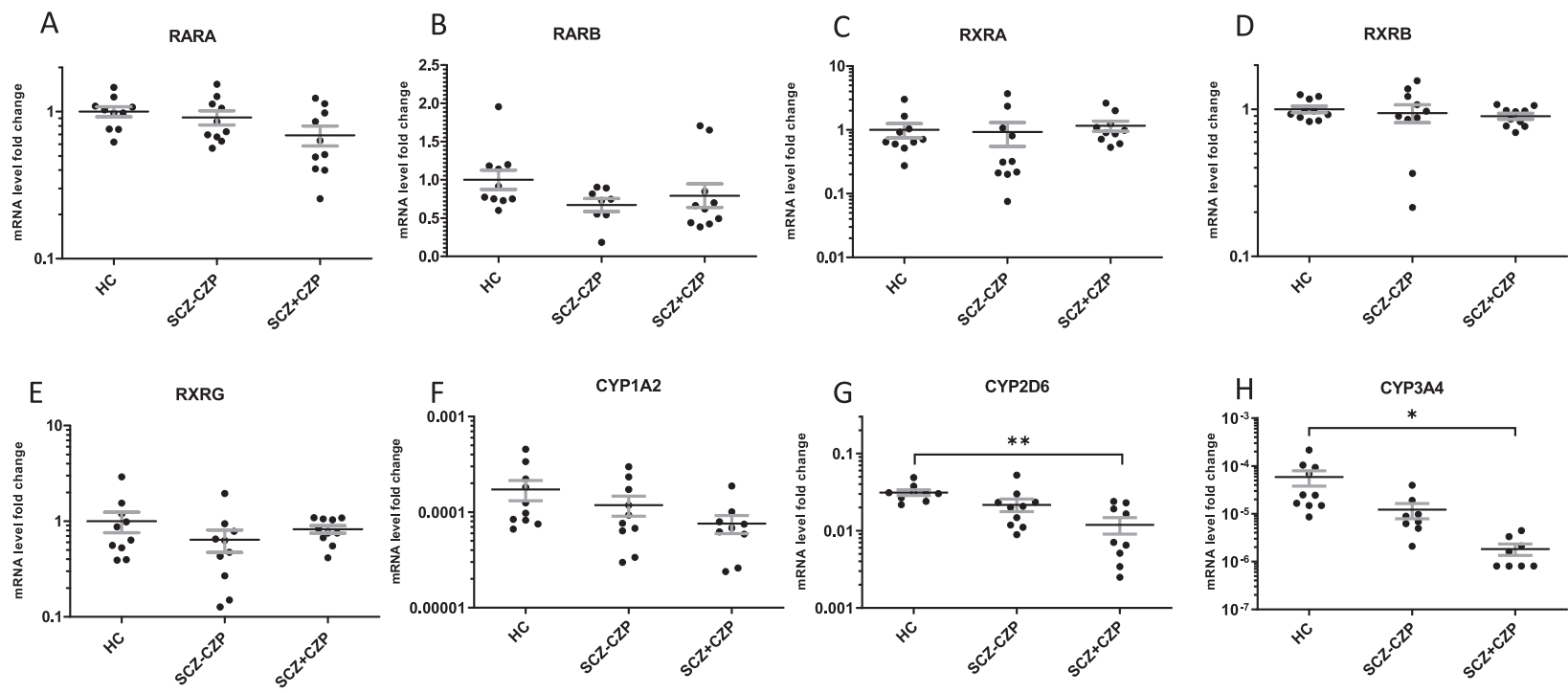

Fig. 5 mRNA Expression of RA Receptors and clozapinemetabolizing CYP450 Isozymes. mRNA transcripts of the main retinoid receptors RARA (a), RARB (b), RXRA (c), RXRB (d) RXRG (e) and clozapine- as well as at-RA metabolizing CYP1A2 (f), CYP2D6 (g) and CYP3A4 (h) were detectable in whole blood-derived mRNA. While there were no main effects of group on any of the five

demonstrated, did not differ between groups [74, 75]. While the expression of RA-inducible RARB, for which associations with schizophrenia have been demonstrated $[21,25]$ did not differ significantly between groups (Fig. 5b), the tendency toward reduced expression in SCZCZP is similar to the pattern observed for CYP26A and STRA6 (Fig. 4). Rather unexpected were findings on reduced CYP2D6 and CYP3A4. On the one hand, reduced expression of non-specific RA-degrading cytochromes may provide a mechanism for increased at-RA serum levels in clozapine-treated subjects. On the other hand, recent in vitro evidence demonstrates the downregulation of CYP2D6 mRNA upon stimulation with retinoids [76], which suggests that reduced levels of CYP2D6 in clozapine patients may also be a consequence of increased RA-signaling. The results must, however, be interpreted with caution, particularly because little is known about the role and functional implications of altered blood-cell-based mRNA expression of CYP450 isozymes.

Limitations of our study include the cross-sectional design and the comparably small sample size of the clinical part. While we were able to show significant inhibition of $a t$-RA catabolism at a functional level in various tissues and patient-specific cells, our methodological approach cannot reveal the relative contribution of the different CYP isoforms to the observed effects, which putatively varies between tissues and individual subjects.

While the small sample size was a result of the large effect size of $1 \mu \mathrm{M}$ clozapine in PBMC-derived microsomes (for details see Supplementary S1), the cross-

receptors (one-way ANOVA), the pattern of RA-inducible RARB is similar to the patterns of STRA6 and CYP26A. Interestingly, both CYP2D6 and CYP3A4 mRNA levels were strikingly decreased in $\mathrm{SCZ}+\mathrm{CZP}$ patients compared with healthy controls $(* P<0.05$, ${ }^{* *} P<0.001$, one-way ANOVA with Newman-Keul's post-hoc test).

sectional design cannot provide evidence for a role of clozapine's RA-modulating effects in its antipsychotic mechanism. Due to methodological limitations, endogenous at-RA metabolites were not detectable in patientderived samples. Thus, we cannot definitely attribute the alterations to either increased catabolism or decreased anabolism in SZ patients. Despite our careful control for age, weight, and smoking status, confounding effects of eating habits or nutritional preferences on at-RA homeostasis that were not assessed in the present design cannot be definitely ruled out. On the other hand, the large effect sizes that we observed in both our in vitro studies and the patient-specific biological endpoints strongly suggest a clinical relevance of our findings.

Moreover, human brain samples served as generic human brain tissue and were not available from multiple regions. Thus, our present study was not able to resolve the observed effects in a region-specific manner, which should undoubtedly be pursued in future studies. Finally, while our assays were able to detect effects of the test compounds on gross RA catabolism in the respective tissues, we were not able to discriminate the degree to which specific CYP450 isoforms may have contributed to the observed effects. Major challenges are the unspecific effects of pharmacological inhibition [66]. Such an in-depth analysis of which subjects, and via which isozymes, clozapine may specifically block RA catabolism should be the subject of future studies.

In conclusion, we have identified clozapine to strikingly impact RA catabolism in human adult brain tissue and 
PBMCs, suggesting a retinoid-related mechanism of action of both clozapine and its two major metabolites. Moreover, we identified dysregulation of retinoid homeostasis in patients with SZ at several levels and we revealed potential effects of clozapine on the latter, suggesting normalization of the deficits as part of clozapine's pleiotropic actions. Our strategy to quantify the impact of clozapine on RA homeostasis parameters in patient-derived material could serve as a versatile and simple tool to assess the patient-specific retinoidergic response to clozapine, which might correlate with later treatment response.

Acknowledgements This study was supported by the German Research Foundation (DFG, Grant no. HE 6939/3-1), the Berlin Institute of Health Clinician Scientist Program to JH-R, and a doctoral scholarship of the Studienstiftung des Deutschen Volkes to LRO. The authors gratefully acknowledge the Netherlands Brain Bank (NBB), Netherlands Institute for Neuroscience, Amsterdam, which provided the human brain tissue. Open access funding provided by Projekt DEAL.

\section{Compliance with ethical standards}

Conflict of interest The authors declare that they have no conflict of interest.

Publisher's note Springer Nature remains neutral with regard to jurisdictional claims in published maps and institutional affiliations.

Open Access This article is licensed under a Creative Commons Attribution 4.0 International License, which permits use, sharing, adaptation, distribution and reproduction in any medium or format, as long as you give appropriate credit to the original author(s) and the source, provide a link to the Creative Commons license, and indicate if changes were made. The images or other third party material in this article are included in the article's Creative Commons license, unless indicated otherwise in a credit line to the material. If material is not included in the article's Creative Commons license and your intended use is not permitted by statutory regulation or exceeds the permitted use, you will need to obtain permission directly from the copyright holder. To view a copy of this license, visit http://creativecommons. org/licenses/by/4.0/.

\section{References}

1. Baldessarini RJ, Frankenburg FR. Clozapine. A novel antipsychotic agent. N Engl J Med. 1991;324:746-54.

2. Dold M, Leucht S. Pharmacotherapy of treatment-resistant schizophrenia: a clinical perspective. Evid Ment health. 2014;17:33-7.

3. Siskind D, McCartney L, Goldschlager R, Kisely S. Clozapine v. first- and second-generation antipsychotics in treatment-refractory schizophrenia: systematic review and meta-analysis. Br J Psychiatry: J Ment Sci. 2016;209:385-92.

4. Howes OD, McCutcheon R, Agid O, de Bartolomeis A, van Beveren NJ, Birnbaum ML, et al. treatment-resistant schizophrenia: treatment response and resistance in psychosis (TRRIP) working group consensus guidelines on diagnosis and terminology. Am J Psychiatry. 2017;174:216-29.

5. Samara MT, Dold M, Gianatsi M, Nikolakopoulou A, Helfer B, Salanti G, et al. Efficacy, acceptability, and tolerability of antipsychotics in treatment-resistant schizophrenia: a network metaanalysis. JAMA Psychiatry. 2016;73:199-210.
6. Leucht S, Cipriani A, Spineli L, Mavridis D, Orey D, Richter F, et al. Comparative efficacy and tolerability of 15 antipsychotic drugs in schizophrenia: a multiple-treatments meta-analysis. Lancet. 2013;382:951-62.

7. Hagger C, Buckley P, Kenny JT, Friedman L, Ubogy D, Meltzer HY. Improvement in cognitive functions and psychiatric symptoms in treatment-refractory schizophrenic patients receiving clozapine. Biol Psychiatry. 1993;34:702-12.

8. Meltzer HY. An overview of the mechanism of action of clozapine. J Clin psychiatry. 1994;55(Suppl B):47-52.

9. Kim DD, Barr AM, Honer WG, Procyshyn RM. Reversal of dopamine supersensitivity as a mechanism of action of clozapine. Psychother Psychosom. 2018;87:306-7.

10. Baig MR, Navaira E, Escamilla MA, Raventos H, Walss-Bass C. Clozapine treatment causes oxidation of proteins involved in energy metabolism in lymphoblastoid cells: a possible mechanism for antipsychotic-induced metabolic alterations. J Psychiatr Pract. 2010;16:325-33.

11. Magliaro BC, Saldanha CJ. Clozapine protects PC-12 cells from death due to oxidative stress induced by hydrogen peroxide via a celltype specific mechanism involving inhibition of extracellular signalregulated kinase phosphorylation. Brain Res. 2009;1283:14-24.

12. Nucifora FC Jr., Mihaljevic M, Lee BJ, Sawa A. Clozapine as a model for antipsychotic development. NeuroTherapeutics: J Am Soc Exp NeuroTherapeutics. 2017;14:750-61.

13. Jiang L, Wu X, Wang S, Chen SH, Zhou H, Wilson B, et al. Clozapine metabolites protect dopaminergic neurons through inhibition of microglial NADPH oxidase. J Neuroinflamm. 2016;13:110.

14. Hu X, Zhou H, Zhang D, Yang S, Qian L, Wu HM, et al. Clozapine protects dopaminergic neurons from inflammation-induced damage by inhibiting microglial overactivation. J NeuroImmune Pharmacol. 2012;7:187-201.

15. Hall J, Trent S, Thomas KL, O’Donovan MC, Owen MJ. Genetic risk for schizophrenia: convergence on synaptic pathways involved in plasticity. Biol Psychiatry. 2015;77:52-58.

16. Hsu YT, Li J, Wu D, Sudhof TC, Chen L. Synaptic retinoic acid receptor signaling mediates mTOR-dependent metaplasticity that controls hippocampal learning. Proc Natl Acad Sci USA. 2019; 116:7113-22.

17. Zhong LR, Chen X, Park E, Sudhof TC, Chen L. Retinoic acid receptor RARalpha-dependent synaptic signaling mediates homeostatic synaptic plasticity at the inhibitory synapses of mouse visual cortex. J Neurosci. 2018;38:10454-66.

18. Aoto J, Nam CI, Poon MM, Ting P, Chen L. Synaptic signaling by all-trans retinoic acid in homeostatic synaptic plasticity. Neuron. 2008;60:308-20.

19. Goodman AB. Retinoid dysregulation may result in abnormal expression of glutamic acid decarboxylase in schizophrenia. Arch Gen psychiatry. 1996;53:653.

20. Goodman AB. Chromosomal locations and modes of action of genes of the retinoid (vitamin A) system support their involvement in the etiology of schizophrenia. Am J Med Genet. 1995;60:335-48.

21. Reay WR, Atkins JR, Quide Y, Carr VJ, Green MJ, Cairns MJ. Polygenic disruption of retinoid signalling in schizophrenia and a severe cognitive deficit subtype. Mol Psychiatry. 2020;25:719-31.

22. Wan C, Yang Y, Li H, La Y, Zhu H, Jiang L, et al. Dysregulation of retinoid transporters expression in body fluids of schizophrenia patients. J Proteome Res. 2006;5:3213-6.

23. Feng J, Chen J, Yan J, Jones IR, Craddock N, Cook EH Jr., et al. Structural variants in the retinoid receptor genes in patients with schizophrenia and other psychiatric diseases. Am J Med Genet Part B Neuropsychiatr Genet. 2005;133B:50-53.

24. Ishiguro H, Okubo Y, Ohtsuki T, Yamakawa-Kobayashi K, Arinami T. Mutation analysis of the retinoid X receptor beta, nuclearrelated receptor 1 , and peroxisome proliferator-activated receptor alpha genes in schizophrenia and alcohol dependence: possible 
haplotype association of nuclear-related receptor 1 gene to alcohol dependence. Am J Med Genet. 2002;114:15-23.

25. Reay WR, Cairns MJ. The role of the retinoids in schizophrenia: genomic and clinical perspectives. Molecular Psychiatry. 2020; 25:706-18.

26. Blomhoff R, Blomhoff HK. Overview of retinoid metabolism and function. J Neurobiol. 2006;66:606-30.

27. Lane MA, Bailey SJ. Role of retinoid signalling in the adult brain. Prog Neurobiol. 2005; 75:275-93.

28. Shearer KD, Stoney PN, Morgan PJ, McCaffery PJ. A vitamin for the brain. Trends Neurosci. 2012;35:733-41.

29. Chiang MY, Misner D, Kempermann G, Schikorski T, Giguere V, Sucov HM, et al. An essential role for retinoid receptors RARbeta and RXRgamma in long-term potentiation and depression. Neuron. 1998;21:1353-61.

30. Arendt KL, Zhang Y, Jurado S, Malenka RC, Sudhof TC, Chen L. Retinoic acid and LTP recruit postsynaptic AMPA receptors using distinct SNARE-dependent mechanisms. Neuron. 2015;86:442-56.

31. Corcoran J, Maden M. Nerve growth factor acts via retinoic acid synthesis to stimulate neurite outgrowth. Nat Neurosci. 1999; 2:307-8.

32. Maghsoodi B, Poon MM, Nam CI, Aoto J, Ting P, Chen L. Retinoic acid regulates RARalpha-mediated control of translation in dendritic RNA granules during homeostatic synaptic plasticity. Proc Natl Acad Sci USA. 2008;105:16015-20.

33. Sarti F, Schroeder J, Aoto J, Chen L. Conditional RARalpha knockout mice reveal acute requirement for retinoic acid and RARalpha in homeostatic plasticity. Front Mol Neurosci. 2012;5:16.

34. Keshavan MS, Mehta UM, Padmanabhan JL, Shah JL. Dysplasticity, metaplasticity, and schizophrenia: Implications for risk, illness, and novel interventions. Dev Psychopathol. 2015;27:615-35.

35. Goodman AB. Three independent lines of evidence suggest retinoids as causal to schizophrenia. Proc Natl Acad Sci USA. 1998;95:7240-4.

36. Rioux L, Arnold SE. The expression of retinoic acid receptor alpha is increased in the granule cells of the dentate gyrus in schizophrenia. Psychiatry Res. 2005;133:13-21.

37. Goodman AB. Microarray results suggest altered transport and lowered synthesis of retinoic acid in schizophrenia. Mol Psychiatry. 2005;10:620-1.

38. Corley SM, Tsai SY, Wilkins MR, Shannon Weickert C. Transcriptomic analysis shows decreased cortical expression of NR4A1, NR4A2 and RXRB in schizophrenia and provides evidence for nuclear receptor dysregulation. PLoS ONE. 2016;11: $\mathrm{e} 0166944$.

39. Samad TA, Krezel W, Chambon P, Borrelli E. Regulation of dopaminergic pathways by retinoids: activation of the $\mathrm{D} 2$ receptor promoter by members of the retinoic acid receptor-retinoid $\mathrm{X}$ receptor family. Proc Natl Acad Sci USA. 1997;94:14349-54.

40. Lerner V, McCaffery PJ, Ritsner MS. Targeting retinoid receptors to treat schizophrenia: rationale and progress to date. CNS Drugs. 2016;30:269-80.

41. Lerner V, Miodownik C, Gibel A, Sirota P, Bush I, Elliot H, et al. The retinoid $\mathrm{X}$ receptor agonist bexarotene relieves positive symptoms of schizophrenia: a 6-week, randomized, double-blind, placebo-controlled multicenter trial. J Clin Psychiatry. 2013;74: 1224-32.

42. Lerner V, Miodownik C, Gibel A, Kovalyonok E, Shleifer T, Goodman $\mathrm{AB}$, et al. Bexarotene as add-on to antipsychotic treatment in schizophrenia patients: a pilot open-label trial. Clin Neuropharmacol. 2008;31:25-33.

43. Stoney PN, Fragoso YD, Saeed RB, Ashton A, Goodman T, Simons C, et al. Expression of the retinoic acid catabolic enzyme CYP26B1 in the human brain to maintain signaling homeostasis. Brain Struct Funct. 2016;221:3315-26.
44. Fragoso YD, Shearer KD, Sementilli A, de Carvalho LV, McCaffery PJ. High expression of retinoic acid receptors and synthetic enzymes in the human hippocampus. Brain Struct Funct. 2012;217:473-83.

45. Maden M. Retinoic acid in the development, regeneration and maintenance of the nervous system. Nat Rev Neurosci. 2007;8: 755-65.

46. Clemens V, Regen F, Le Bret N, Heuser I, Hellmann-Regen J. Anti-inflammatory effects of minocycline are mediated by retinoid signaling. BMC Neurosci. 2018;19:58.

47. Clemens V, Regen F, Le Bret N, Heuser I, Hellmann-Regen J. Retinoic acid enhances apolipoprotein $\mathrm{E}$ synthesis in human macrophages. J Alzheimer's Dis. 2018;61:1295-1300.

48. Regen F, Le Bret N, Hildebrand M, Herzog I, Heuser I, HellmannRegen J. Inhibition of brain retinoic acid catabolism: a mechanism for minocycline's pleiotropic actions? World J Biol Psychiatry. 2016;17:634-40.

49. Regen F, Herzog I, Hahn E, Ruehl C, Le Bret N, Dettling M, et al. Clozapine-induced agranulocytosis: Evidence for an immunemediated mechanism from a patient-specific in-vitro approach. Toxicol Appl Pharmacol. 2017;316:10-16.

50. Regen F, Hildebrand M, Le Bret N, Herzog I, Heuser I, Hellmann-Regen J. Inhibition of retinoic acid catabolism by minocycline: evidence for a novel mode of action? Exp Dermatol. 2015;24:473-6.

51. Hellmann-Regen J, Uhlemann R, Regen F, Heuser I, Otte C, Endres M, et al. Direct inhibition of retinoic acid catabolism by fluoxetine. J Neural Transm. 2015;122:1329-38.

52. Regen F, Le Bret N, Hildebrand M, Herzog I, Heuser I, HellmannRegen J. Inhibition of brain retinoic acid catabolism: a mechanism for minocycline's pleiotropic actions? World J Biol Psychiatry. 2016;17:634-40.

53. Ye J, Coulouris G, Zaretskaya I, Cutcutache I, Rozen S, Madden TL. Primer-BLAST: a tool to design target-specific primers for polymerase chain reaction. BMC Bioinforma. 2012;13:134.

54. Baldessarini RJ, Centorrino F, Flood JG, Volpicelli SA, HustonLyons D, Cohen BM. Tissue concentrations of clozapine and its metabolites in the rat. Neuropsychopharmacology. 1993;9: 117-24.

55. Wilk S, Stanley M. Clozapine concentrations in brain regions: relationship to dopamine metabolite increase. Eur J Pharmacol. 1978;51:101-7.

56. Hiemke C, Bergemann N, Clement HW, Conca A, Deckert J, Domschke $\mathrm{K}$, et al. Consensus guidelines for therapeutic drug monitoring in neuropsychopharmacology: update 2017. Pharmacopsychiatry. 2018;51:9-62.

57. Hyttel J, Overo KF, Arnt J. Biochemical effects and drug levels in rats after long-term treatment with the specific 5-HT-uptake inhibitor, citalopram. Psychopharmacology. 1984;83:20-27.

58. Wang JS, DeVane CL, Gibson BB, Donovan JL, Markowitz JS, Zhu HJ. Population pharmacokinetic analysis of drug-drug interactions among risperidone, bupropion, and sertraline in CF1 mice. Psychopharmacology. 2006;183:490-9.

59. Wong PT, Yoong YL, Gwee MC. Marked variation in diazepam sensitivity in Swiss albino mice. Life Sci. 1986;39:945-52.

60. Nedahl M, Johansen SS, Linnet K. Reference brain/blood concentrations of citalopram, duloxetine, mirtazapine and sertraline. J Anal Toxicol. 2018;42:149-56.

61. Skov L, Holm KM, Johansen SS, Linnet K. Postmortem brain and blood reference concentrations of alprazolam, bromazepam, chlordiazepoxide, diazepam, and their metabolites and a review of the literature. J Anal Toxicol. 2016;40:529-36.

62. Takamura R, Watamura N, Nikkuni M, Ohshima T. All-trans retinoic acid improved impaired proliferation of neural stem cells and suppressed microglial activation in the hippocampus in an Alzheimer's mouse model. J Neurosci Res. 2017;95:897-906. 
63. Dheen ST, Jun Y, Yan Z, Tay SS, Ling EA. Retinoic acid inhibits expression of TNF-alpha and iNOS in activated rat microglia. Glia. 2005;50:21-31.

64. Forsyth JK, Lewis DA. Mapping the consequences of impaired synaptic plasticity in schizophrenia through development: an integrative model for diverse clinical features. Trends Cogn Sci. 2017;21:760-78.

65. Kedishvili NY. Retinoic acid synthesis and degradation. Subcell Biochem. 2016;81:127-61.

66. Thatcher JE, Isoherranen $\mathrm{N}$. The role of CYP26 enzymes in retinoic acid clearance. Expert Opin drug Metab Toxicol. 2009;5: 875-86.

67. Regen F, Heuser I, Herzog I, Hellmann-Regen J. Striking growthinhibitory effects of minocycline on human prostate cancer cell lines. Urology. 2014;83:509. e501-509. e506

68. Zhang L, Zheng H, Wu R, Kosten TR, Zhang XY, Zhao J. The effect of minocycline on amelioration of cognitive deficits and pro-inflammatory cytokines levels in patients with schizophrenia. Schizophrenia Res. 2019;212:92-98.

69. Zhang L, Zheng H, Wu R, Zhu F, Kosten TR, Zhang XY, et al. Minocycline adjunctive treatment to risperidone for negative symptoms in schizophrenia: association with pro-inflammatory cytokine levels. Prog Neuro-Psychopharmacol Biol Psychiatry. 2018;85:69-76.
70. Miyaoka T, Yasukawa R, Yasuda H, Hayashida M, Inagaki T, Horiguchi J. Possible antipsychotic effects of minocycline in patients with schizophrenia. Prog Neuro-Psychopharmacol Biol Psychiatry. 2007;31:304-7.

71. Xiong Z, Cheng M, Zhu P, Huang S, Guo J, Zhang W, et al. Association of blood cell counts with the risk of olanzapine- or clozapine-induced dyslipidemia in Chinese schizophrenia patients. Hum Psychopharmacol. 2019;34:e2699.

72. Lilley JS, Linton MF, Fazio S. Oral retinoids and plasma lipids. Dermatol Ther. 2013;26:404-10.

73. Napoli JL. Quantification of physiological levels of retinoic acid. Methods Enzymol. 1986;123:112-24.

74. Chan RF, Shabalin AA, Montano C, Hannon E, Hultman CM, Fallin MD et al. Independent methylome-wide association studies of schizophrenia detect consistent case-control differences. Schizophrenia Bull. 2019.

75. Wan C, Shi Y, Zhao X, Tang W, Zhang M, Ji B, et al. Positive association between ALDH1A2 and schizophrenia in the Chinese population. Prog Neuro-Psychopharmacol Biol Psychiatry. 2009;33:1491-5.

76. Stevison F, Kosaka M, Kenny JR, Wong S, Hogarth C, Amory $\mathrm{JK}$, et al. Does in vitro cytochrome $\mathrm{P} 450$ downregulation translate to in vivo drug-drug interactions? Preclinical and clinical studies with 13-cis-retinoic acid. Clin Transl Sci. 2019;12:350-60. 